\title{
Mapping Aerosolized Saliva on Face Coverings for Biosensing Applications
}

Zhicheng Jin, ${ }^{\mathrm{a}, \dagger}{ }^{\dagger}$ Alec Jorns,,${ }^{\mathrm{a},} \dagger$ Wonjun Yim, ${ }^{\mathrm{b}}$ Ryan Wing, ${ }^{\mathrm{a}}$ Yash Mantri, ${ }^{\mathrm{c}}$ Jiajing Zhou, ${ }^{\mathrm{a}}$ Jingcheng

Zhou, ${ }^{a}$ Zhuohong Wu, ${ }^{a}$ Colman A. Moore, ${ }^{a}$ William Penny, ${ }^{d}$ Jesse V. Jokerst ${ }^{\mathrm{a}, \mathrm{b}, \mathrm{e}^{*}}$

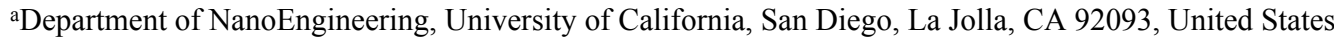
bMaterials Science and Engineering Program, University of California, San Diego, La Jolla, CA 92093, United States

'Department of Bioengineering, University of California San Diego, La Jolla, CA 92093, United States

dDivision of Cardiology, VA San Diego Healthcare System, San Diego, CA 92161, United States

eDepartment of Radiology, University of California, San Diego, La Jolla, CA 92093, United States

*Corresponding author's E-mail: jiokerst@ucsd.edu (J.V.J.)

\section{Table of Contents}

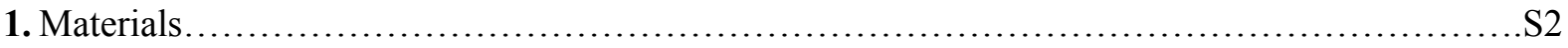

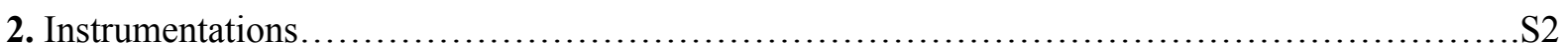

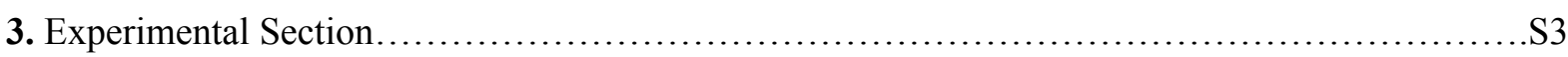

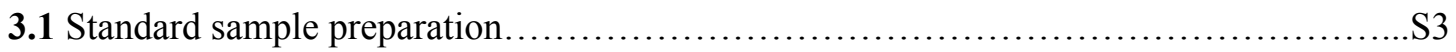

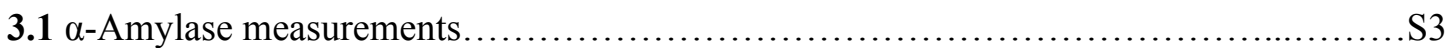

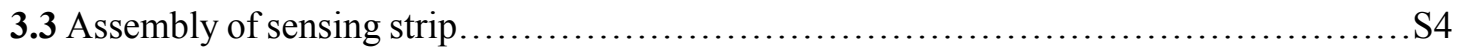

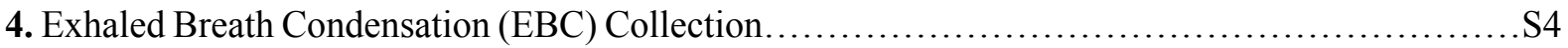

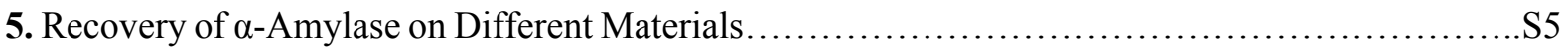

Table S1. The measured activities $(\mathrm{U} / \mathrm{L})$ and distribution $\left(\mathrm{cm}^{2}\right.$ and $\left.\mathrm{I} / \mathrm{cm}^{2}\right)$ of saliva aerosols on various

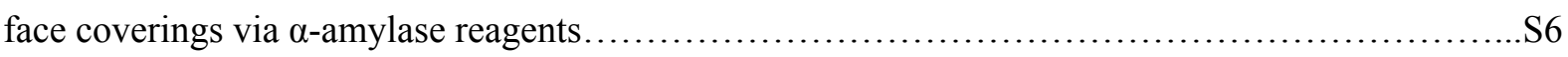

Figure S1. The appearances and corresponding SEM images of outer, middle, and inner layer of face

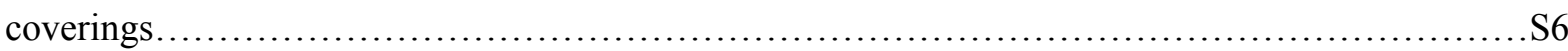

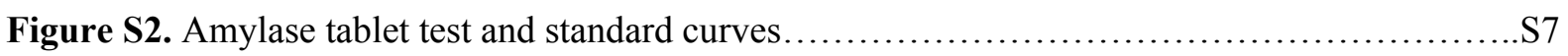

Figure S3. A lab-based condensate set-up for collecting exhaled breath condensation (EBC).........S7

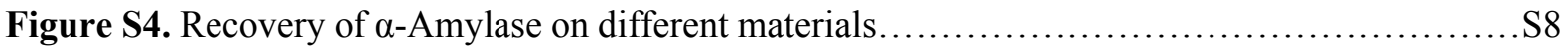




\section{Materials}

Surgical protection masks (made of polypropylene) were purchased from Albatross MFG (Taunton, MA), cloth masks (made of 100\% cotton) were purchased from Port Authority Clothing manufacture, neck gaiters (made of $90 \%$ polyester and $10 \%$ spandex) were purchased from Anstronic manufacture, and Kimtech N95 Pouch Respirators (model 53358, made of polypropylene, NIOSH-approved) were purchased from Kimberly-Clark. Phadebas ${ }^{\circledR}$ Amylase test (50 tablets) and Forensic A4 were purchased from Thermo Fisher Scientific (Magle Life Sciences, Lund, Sweden). The $\alpha$-amylase from human saliva ( $\geq 2 \mathrm{kU} / \mathrm{mL}$ ) and pooled whole human saliva were purchased from Lee Biosolutions, Inc (Maryland Heights, MO). The whole human saliva from each tested subject were also collected through passive drool. The exhaled breath condensation (EBC) was collected from tested subjects using a lab condensate tube setup. Ultrapure water $(18 \mathrm{M} \Omega \cdot \mathrm{cm})$ was obtained from a Milli-Q Integral 5 system (Thermo Fisher Scientific, PA). Amylase sensing strip adhesives Facemount ${ }^{\circledR}$ (P1) and MultiTac ${ }^{\mathrm{TM}}$ (P6) were procured online from Drytac. Clear Duralar ${ }^{\mathrm{TM}}$ polyester film (P2) was purchased from Grafix Plastics for use as a channel spacer. The $6 \mathrm{~mm}$ thermoformed blister packs (P5) were recovered from a 25 -serving sheet of Dulcolax ${ }^{\circledR}$ Bisacodyl $5 \mathrm{mg}$ tablets, purchased in a 100-tablet pack from Amazon. The absorbent pads (P3) were In Vitro Diagnostic (IVD) materials obtained from Ahlstrom Munksjo. The absorbent pad materials used include grades 8950, 6613H, Cytosep 1660, and 601, with compositions of chopped glass with binder, treated spunbound polyester, proprietary fiber blend, and cotton linter fibers, respectively. No unexpected or unusually high safety hazards were encountered.

\section{Instrumentations}

The absorbance at $620 \mathrm{~nm}$ was readout in a hybrid multi-mode microplate reader (model Synergy $^{\mathrm{TM}} \mathrm{H} 1$, BioTek Instruments, Inc.) using $360 \mu \mathrm{L}$ of solutions in 96 -well plates. A scanning electron microscope (SEM, FEI Apreo) was used to analyze the structural information of fibers in all face coverings. SEM images were taken at an accelerating voltage of $1 \mathrm{kV}$ and a current of $0.10 \mathrm{nA}$. A Cricut Explore Air ${ }^{\circledR} 2$ vinyl cutter (Cricut, Inc.) was used to customize the pieces for assembling the portable sensing strip. 


\section{Experimental Section}

3.1 Standard sample preparation. The $\alpha$-amylase standards of $200 \mu \mathrm{L}$ (known stock concentration $\geq 2 \mathrm{kU} / \mathrm{mL}$, human saliva source, Lee Biosolutions, Inc.) were prepared at the following dilutions (using DI water) for standard curve construction: 1/100, 1/125, 1/250, 1/500, $1 / 1000,1 / 2500,1 / 5000,1 / 10,000$, and 1/20,000. Similarly, the pooled whole human saliva samples of $200 \mu \mathrm{L}$ (Lee Biosolutions, Inc.) were prepared at the following dilutions using DI water for standard curve construction: 1/2,1/8,1/16,1/20,1/40,1/100,1/200,1/400, and 1/2000. The saliva and exhaled breath condensate matrixes from each participant were collected, spun down, and the supernatant of $10 \mu \mathrm{L}$ was retrieved for amylase reagent test.

3.2 $\alpha$-Amylase measurements. The amount and distribution of $\alpha$-amylase on the face coverings or strips were measured using amylase tablet reagent and forensic paper (Phadebas, Inc.). The amylase-sensitive reagent could detect the amylase activities as low as $200 \mathrm{U} / \mathrm{L}$ and was used on scales of $180 \mathrm{mg}$ and $18 \mathrm{mg}$ to assess the activity of amylase present on the face coverings. No unexpected or unusually high safety hazards were encountered.

(i) Detection on face coverings. Briefly, a circle area of $\sim 20 \mathrm{~cm}^{2}$ of interest was excised from the face coverings (all layers) and cut into small pieces. In a $20 \mathrm{~mL}$-scintillation vial, the mask pieces were loaded, and $4.2 \mathrm{~mL}$ of DI water was added. The mixture was vortexed and incubated at $37^{\circ} \mathrm{C}$ water bath for $5 \mathrm{~min}$. After incubation, the supernatant was extracted from the above mixture into a $15-\mathrm{mL}$ Falcon tube by passing the mixture though a syringe. An amylase tablet was added into the saliva extraction and the tested sample was vortexed for 10 sec, appearing as blue suspension. The reaction was further incubated at $37{ }^{\circ} \mathrm{C}$ for $15 \mathrm{~min}$ and stopped by adding $1 \mathrm{~mL}$ of $0.5 \mathrm{M} \mathrm{NaOH}$ solution. The suspension was then centrifuged at $1,500 \mathrm{~g}$ for $5 \mathrm{~min}$ and the optical density of supernatant at $620 \mathrm{~nm}$ was measured. Note that for all the tested samples, a dilution factor of 18 was applied for the optical absorbance reading. The standard curve of $\alpha$-amylase activities, the amount of $\alpha$-amylase in saliva samples from each participant, and captured amylase levels on various face coverings were tested using above procedures.

(ii) Detection on sensing strips. Briefly, $\sim 0.72 \mathrm{~cm}^{2}$ of face covering or strip pad of interest was excised; $420 \mu \mathrm{L}$ of DI water was added and the mixture was incubated at $37{ }^{\circ} \mathrm{C}$ for $5 \mathrm{~min}$. Next, a $18 \mathrm{mg}$ amylase tablet portion was added and vortexed. The reaction was then incubated at 37 ${ }^{\circ} \mathrm{C}$ for $15 \mathrm{~min}$ and quenched by $100 \mu \mathrm{L}$ of $0.5 \mathrm{M} \mathrm{NaOH}$ solution. The work up and absorbance 
measurements followed the same protocol, as above.

(iii) Distribution detection. Briefly, the face covering of interest was laid flat on a clean benchtop. DI water was sprayed evenly on the flattened mask to fully soak the mask materials. Depending on the materials wettability and water absorption ability, the volume of sprayed DI water can vary from 5 to $10 \mathrm{~mL}$. Next, a forensic press paper was covered on the top of wet mask with the blue side directly contacting to the mask materials. A second round of DI water ( 2-3 mL) was sprayed on the forensic press paper, and the system was then secured with a clean and heavy glass. The reaction at the interface was allowed for $40 \mathrm{~min}$ incubation before drying the paper. This distribution paper was then laid flat and imaged by smartphone inside a professional lightbox photo studio. Each distribution was measured with three replicates. The amylase-presenting area and mean gray value of the distribution replicates were analyzed in ImageJ software using the built-in functions, e.g., merge channels, make grid, and measure area and mean gray value (i.e., color intensity per $\mathrm{cm}^{2}$ ). The determined color intensities were plotted as a heat map in Excel and a scale bar of 0-270 I/ $\mathrm{cm}^{2}$ was used, with the color intensities above $90 \mathrm{I} / \mathrm{cm}^{2}$ appearing as gradient blue.

3.3 Assembly of sensing strip. The portable strip device was composed of several thin materials, custom-cut using a Cricut Explore Air $^{\circledR} 2$ vinyl cutter. The top cover (P1) was a vinyl adhesive that acted as a mounting surface for the channel spacer (P2). An absorbent pad (P3), composed of spunbound polyester was placed onto the cover in the precut spacer channels for concentrating the transmitted aerosolized saliva. To assemble the standard sensing strip, first we removed the release liner from the bottom of the top cover, exposing the acrylic adhesive. Then, the channel spacer was aligned and placed on the exposed cover adhesive. Next, the blister pack (P5) was set into the precut hole and filled with a specific reagent. The absorbent pad decorated with specific reagents was laid into the exposed adhesive within the precut channels in the channel spacer. Finally, the release liner was removed from the top of the bottom adhesive layer (P6) exposing the adhesive, and this layer was aligned and placed to the above structure to seal the entire device. After all these components were placed, they were pressed firmly to remove any air bubbles. The assembly of amylase-sensing strip required an additional layer of precut forensic press paper (P4) sandwiched underneath the absorbent pad.

\section{Exhaled Breath Condensation (EBC) Collection}


A lab-based set-up for EBC collection was illustrated in Figure S3. Ice water was first loaded into the condensate tube, and the top adaptor was seal with cotton to avoid atmosphere air condensation. The bottom of condensate tube was connected to a two-neck round bottom flask for EBC collection. The second neck of the flask was mount with a three-way valve for breath vent. The large saliva droplets from the tested subjects are mostly stopped and trapped by the three-way valve, which only allows the breath condensate pass through. The subject keeps breathing for 5-10 min through the valve yields about $1 \mathrm{~mL}$ of EBC solution. The obtained EBC solutions were frozen at $-80{ }^{\circ} \mathrm{C}$ for storage.

\section{Recovery of $\alpha$-Amylase on Different Materials}

The pooled whole human saliva was diluted by a factor of 5 before use. To simulate a worn face covering contaminated with saliva aerosols, $5 \mu \mathrm{L}$ of the above diluted sample was pipetted onto different materials $\left(\sim 2 \times 0.5 \mathrm{~cm}^{2}\right)$, including sized mask of 4 types (e.g., clean neck gaiter, cloth mask, surgical mask, and N95 respirator) and clean absorbent pad of 4 types (S1: Cytosep 1660 cellulose-based fiber, S2: 8950 glass fibers, S3: 601 cellulose-based cotton, and S4: $6613 \mathrm{H}$ spunbound polyester). The saliva on the absorbent pads and face coverings was dried for 8 hours at ambient conditions. Next, each material was placed into a 2-mL microfuge tube; $420 \mu \mathrm{L}$ of DI water was added to above tube and incubated at $37^{\circ} \mathrm{C}$ for $5 \mathrm{~min}$. Next, an aliquot of the tablet was added and vortexed for $10 \mathrm{sec}$. The reaction was further incubated at $37{ }^{\circ} \mathrm{C}$ for $15 \mathrm{~min}$ and stopped by adding $100 \mu \mathrm{L}$ of $0.5 \mathrm{M} \mathrm{NaOH}$ solution. The suspension was then centrifuged at $1,500 \mathrm{~g}$ for $5 \mathrm{~min}$ and the optical density of supernatant at $620 \mathrm{~nm}$ was measured.

The above diluted pooled saliva sample $(5 \mu \mathrm{L})$ was loaded and dried on the clean neck gaiter $\left(\sim 2 \times 0.5 \mathrm{~cm}^{2}\right)$, treated under one of the below conditions, and tested with aliquoted amylase tablet reagent. The used conditions include: (1) sitting for 12 hours at room temperature; (2) exposing to UV radiation $\left(\lambda=365 \mathrm{~nm}\right.$ at $\left.1.36 \mathrm{~mW} / \mathrm{cm}^{2}\right)$ for $30 \mathrm{~min}$; (3) dry heating to $70{ }^{\circ} \mathrm{C}$ for $30 \mathrm{~min}$ for each cycle; (4) spraying with $70 \%$ alcohol 3 times; (5) microwaving at $1200 \mathrm{~W}$ for $5 \mathrm{~min}$; (6) passing the rinsed mask solution through a $0.22 \mu \mathrm{m}$ sterile filter; (7) rinsing the mask materials with DI water 3 times; (8) rinsing the mask materials with $0.5 \mathrm{M} \mathrm{NaOH}$ solution. 
Table S1. The measured activities (U/L) and distribution $\left(\mathrm{cm}^{2}\right.$ and $\left.\mathrm{I} / \mathrm{cm}^{2}\right)$ of saliva aerosols on various face coverings via $\alpha$-amylase reagents.

\begin{tabular}{|c|c|c|c|c|c|}
\hline & \multicolumn{2}{|c|}{ Amylase tablet reagent } & \multicolumn{2}{|c|}{ Forensic press paper } \\
\hline & & $A b s_{\text {me }}$ (a.u.) & Activity (U/L) & Area $\left(\mathrm{cm}^{2}\right)$ & $\begin{array}{c}\text { Intensity } \\
\left(\mathrm{I} / \mathrm{cm}^{2}\right)\end{array}$ \\
\hline \multirow{4}{*}{ Type } & Neck gaiter & 16.8 & $\geq 4,000$ & 45 & 182 \\
\hline & Cloth mask & 4.9 & 1,400 & 25 & 169 \\
\hline & Surgical mask & 10.3 & 3,200 & 56 & 196 \\
\hline & N95 respirator & 3.1 & 300 & 156 & 83 \\
\hline \multirow{3}{*}{$\begin{array}{c}\text { Layer } \\
\text { (surgical mask) }\end{array}$} & Outer & 0.7 & $\leq 200$ & 42 & 193 \\
\hline & Middle & 0.7 & $\leq 200$ & 10 & 31 \\
\hline & Inner & 9.3 & 2,866 & 0.5 & 4 \\
\hline \multirow{4}{*}{$\begin{array}{c}\text { Time } \\
\text { (neck gaiter) }\end{array}$} & $1 \mathrm{~h}$ & 2 & 433 & 4 & 20 \\
\hline & $4 \mathrm{~h}$ & 9.7 & 3,000 & 12 & 40 \\
\hline & $8 \mathrm{~h}$ & 16.8 & $\geq 4,000$ & 34 & 95 \\
\hline & $16 \mathrm{~h}$ & 14.2 & $\geq 4,000$ & 56 & 151 \\
\hline \multirow{3}{*}{$\begin{array}{c}\text { Activity } \\
\text { (neck gaiter) }\end{array}$} & Breath & 0.7 & $\leq 200$ & - & - \\
\hline & Talk & 10.9 & 3,400 & - & - \\
\hline & Run & 16.1 & $\geq 4,000$ & - & - \\
\hline
\end{tabular}
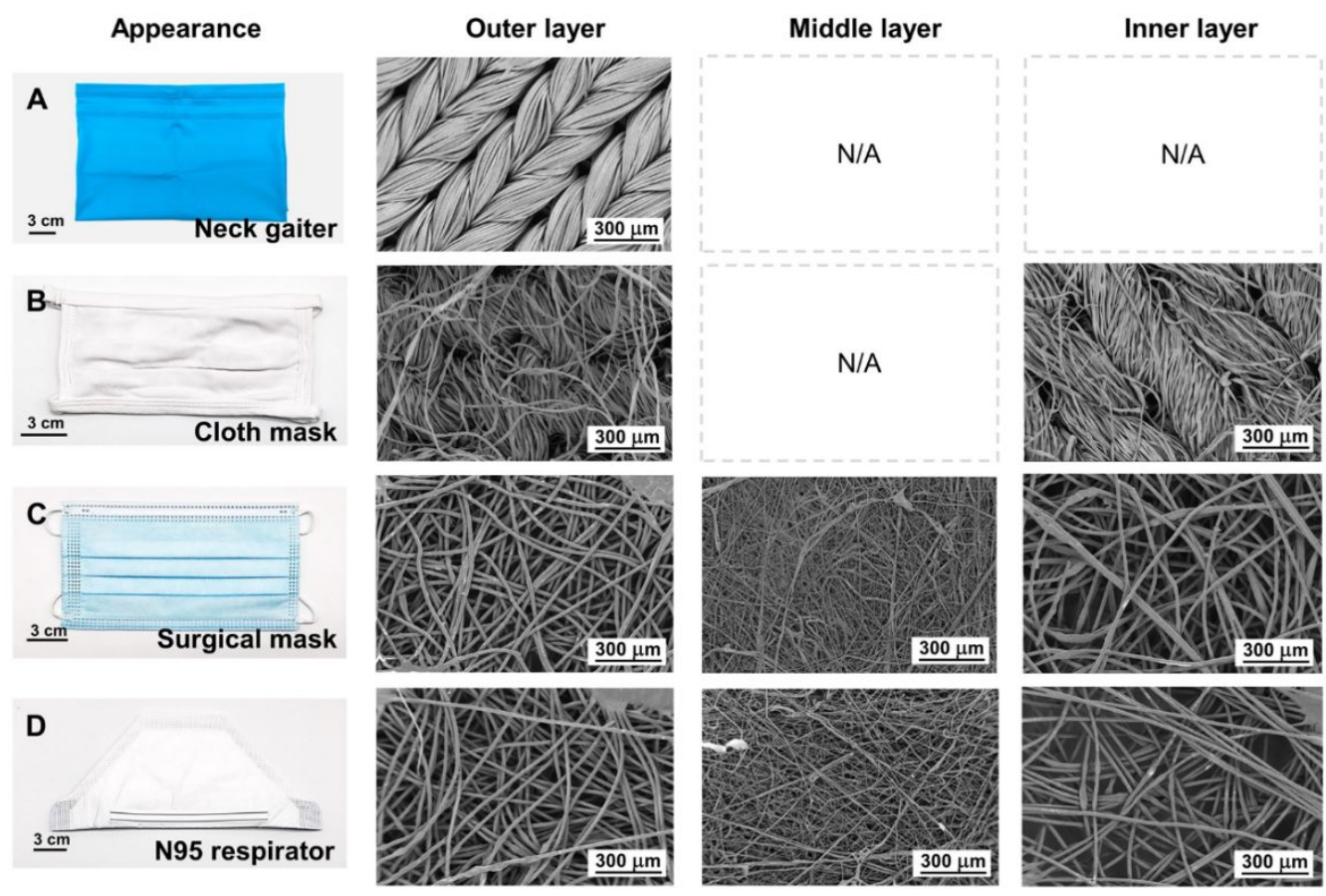

Figure S1. The appearances and corresponding SEM images of outer, middle, and inner layer of face coverings, including (A) neck gaiter, (B) cloth mask, (C) surgical mask, and (D) N95 respirator. "N/A" represents that the SEM image is not measured. In woven neck gaiter and cloth mask, the twisted fibers were organized in parallel and are close to each other. Both masks were highly elastic and stretchable. In comparison, the nonwoven-structured surgical mask and N95 respirator showed randomly crosslinked fiber networks with spunbond materials that were melted together. The middle filtration fabrics of the surgical and N95 face coverings have the smallest pore size ( 260 vs. 3,084 $\mu \mathrm{m}^{2}$ on average, using ImageJ software) compared to other layers. 

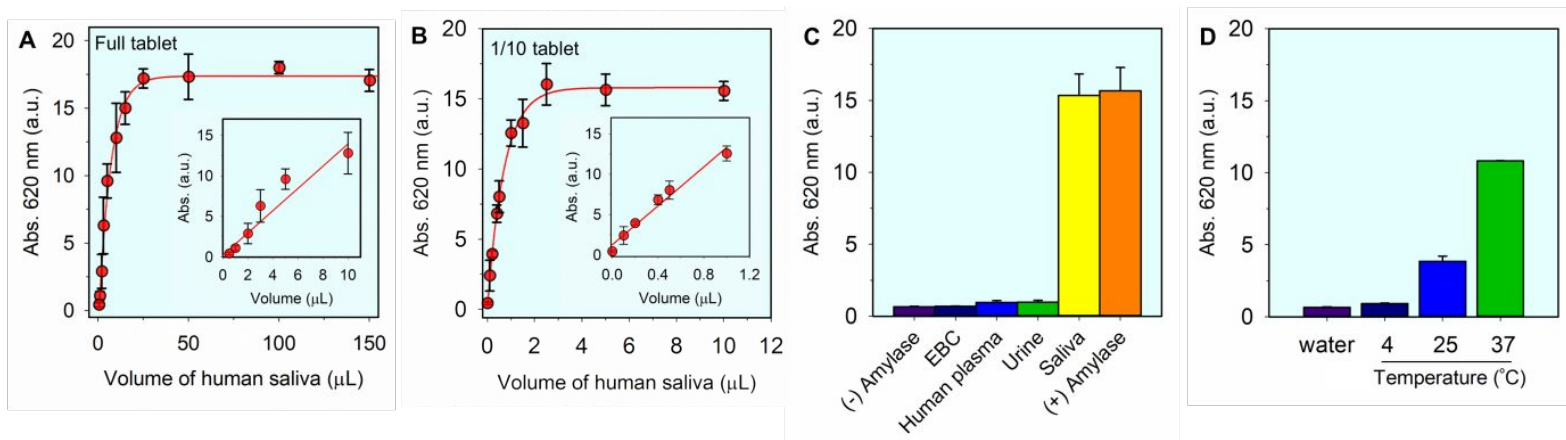

Figure S2. Amylase tablet test and standard curves. (A-B) The standard curve correlates the volume of pooled human saliva to the optical absorbance at $620 \mathrm{~nm}$. Inset shows a linear response was found when the absorbance is below 15. A full amylase tablet (180 mg) was used for the assays in panel $\mathrm{A}$, while an aliquot amylase tablet $(18 \mathrm{mg})$ was used for the assays in panel B. (C) Amylase tablets test (1/10 scale) on various bio-relevant media $(20 \mu \mathrm{L})$, including EBC, human plasma, urine, and saliva. The negative test is water only without $\alpha$-amylase; while the positive control contains commercial $\alpha$-amylase. Only the saliva matrix showed significant signals upon amylase testing. (D) Amylase tablet tests performed on pooled saliva at three temperatures: 4,25 , and $37^{\circ} \mathrm{C} .37^{\circ} \mathrm{C}$ is the optimal condition for producing the most profound color change.
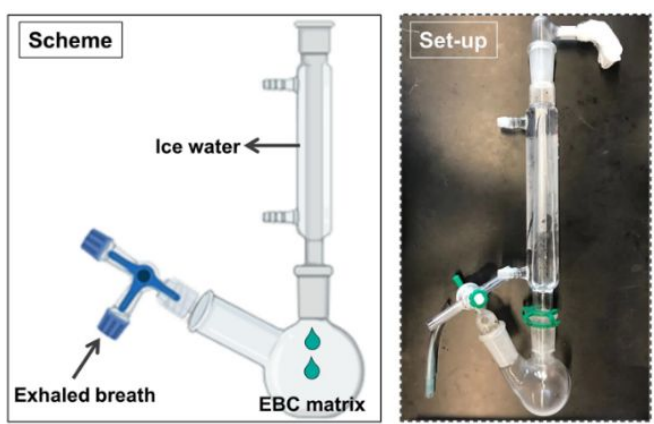

Figure S3. A lab-based condensate set-up for collecting the exhaled breath condensation (EBC) matrix. The participants kept breathing for $\sim 5-10$ min through the valve, which yielded about $1 \mathrm{~mL}$ of EBC sample. 

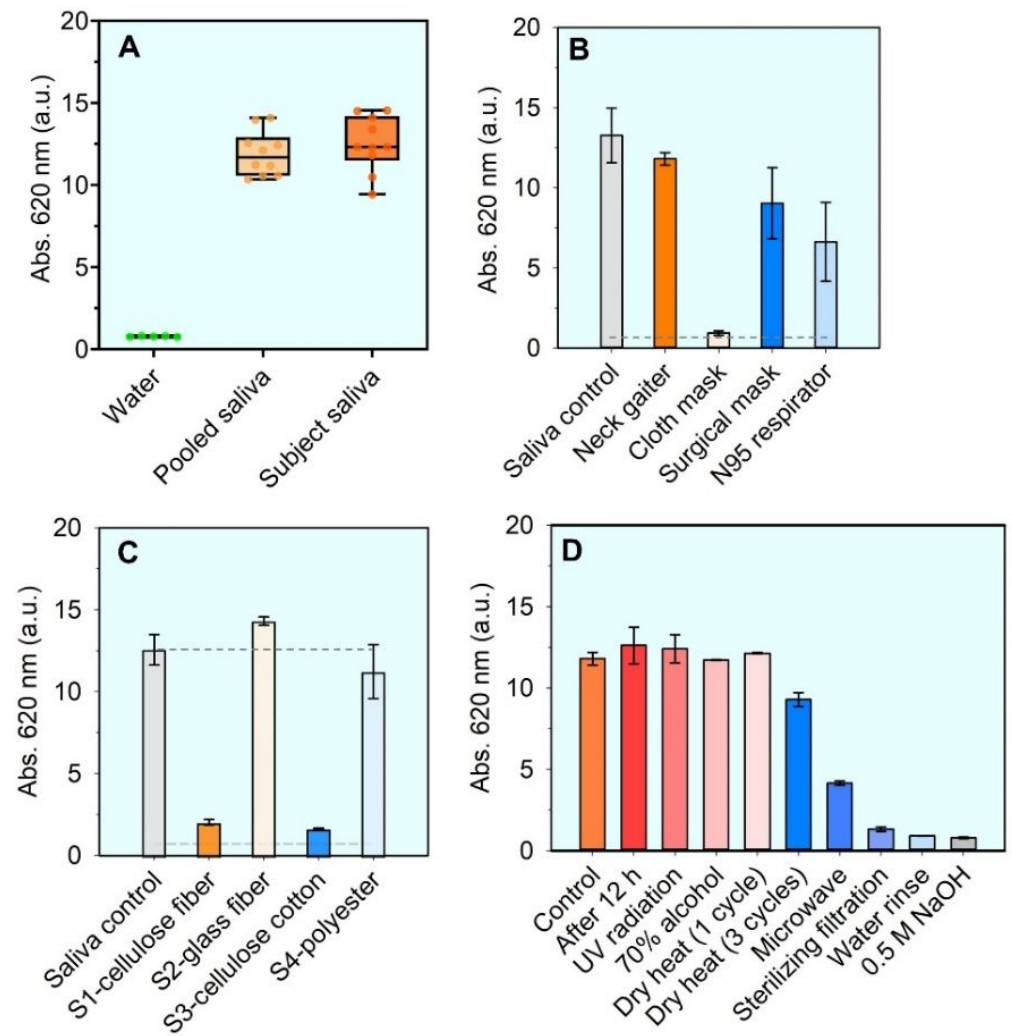

Figure S4. Recovery of $\alpha$-Amylase on different materials. (A) The $\alpha$-amylase level human saliva $(10 \mu \mathrm{L})$ was similar to the one from commercial pooled saliva $(\mathrm{n}=10, p=0.53)$. (B) The recovery/stability of $\alpha$-amylase on four face coverings after $8 \mathrm{~h}$ shows that the cloth mask (made of cellulose) inhibited the amylase activity and only $10 \%$ was recovered in active. The amylase on neck gaiters, surgical mask, and N95 respirator was recovered about 90\%, 70\%, and $50 \%$, respectively. The dashed lines designated the amylase level in saliva (upper) and water (bottom) control. (C) The recovery/stability of $\alpha$-amylase on four absorbent pad materials after $8 \mathrm{~h}$ shows that both Cytosep 1660 (S1, made of proprietary fiber blend, cellulose-based) and 601 (S3, made of cotton linter fibers, cellulose-based) inhibited the amylase activities and the recovery rate was $\sim 10-15 \%$. The amylase loaded on the grades 8950 (S2, made of glass fibers with binder), $6613 \mathrm{H}$ (S4, made of spunbound polyester) remained about $100 \%$ and $90 \%$ active, respectively. The dashed lines designated the amylase level in saliva (upper) and water (bottom) control. (D) The stability of amylase under different environmental treatments, including overnight storage, UV-irradiation, $70 \%$ alcohol, cycles of dry heat at $70{ }^{\circ} \mathrm{C}$, microwave, syringe filtration, $0.5 \mathrm{M} \mathrm{NaOH}$ treatment, and water wash. The amylase remained its bioactivity across many conditions, including overnight storage. Water-washing and base treatment were the most effective means to decontaminate/deactivate the enzymatic activity. 